\title{
Herramientas para la evaluación de la calidad urbana de polígonos de vivienda masiva. Una aproximación empírica a través de 28 polígonos españoles.
}

\author{
Sergio García-Pérez ${ }^{1}$, Javier Monclús ${ }^{2}$, Carmen Díez Medina ${ }^{3}$ \\ U.P. Arquitectura, Universidad de Zaragoza. \\ E-mail: 'sgarciap@unizar.es, 2jmonclus@unizar.es, ${ }^{3}$ cdiezme@unizar.es,
}

\begin{abstract}
Resumen. A pesar de la confianza que el urbanismo funcionalista depositó en los espacios libres de la ciudad de 'torres y bloques', cuestionar aquella visión optimista resulta hoy obligado. Cincuenta años después de la construcción de la mayoría de polígonos modernos de vivienda masiva, la configuración de los espacios abiertos ha demostrado tener una responsabilidad ineludible en lo que se refiere a la calidad urbana de estos conjuntos. Como respuesta a los retos que plantea este importante legado urbano, en el marco del proyecto de investigación UR-Hesp se ha desarrollado una metodología de análisis que trata de evaluar la calidad urbana de los espacios libres de los polígonos residenciales. La aplicación de esta metodología permite identificar puntos fuertes y débiles, así como comparar su calidad en el momento en el que se proyectaron y en la actualidad. Cuestiones como la integración, la permeabilidad, la estructura viaria, la diversidad de usos, la tipología del espacio intermedio, la estructura parcelaria, la densidad, la diversidad edificatoria y la atención por el diseño a la 'altura de los ojos', son evaluadas en detalle para cada caso especifico. El objetivo de esta comunicación es presentar los resultados de la aplicación de dicha metodología de forma pormenorizada a 28 conjuntos de vivienda de Madrid, Barcelona y Zaragoza, así como su potencial extrapolación a otros casos de estudio.
\end{abstract}

Palabras clave: Análisis urbano, diseño urbano, calidad urbana, espacio abierto, España

\section{Introducción}

Tanto la agenda urbana como la política institucional reciente apuesta -sobre todo en los países desarrollados-por centrar la atención sobre la ciudad ya construida, con especial atención sobre las áreas más degradadas (Ministerio de Fomento 2019; United Nations 2015; EU Ministers for Urban Development 2010). Al respecto, muchos polígonos de vivienda han experimentado ciclos de declive, al desarrollar problemas de origen sistémico donde la dimensión física, social y económica está fuertemente interrelacionada. Entre todos ellos, diversas investigaciones han demostrado que los espacios libres juegan un papel relevante en la obsolescencia -o resiliencia-de estos tejidos urbanos, con claras oportunidades de mejora (García-Pérez 2017; Monclús, Díez Medina, y García-Pérez 2017; Sotoca 2012). Además, frente a la aparente homogeneidad que caracterizó inicialmente la aplicación de los principios funcionalistas, cincuenta años después de su construcción los polígonos de vivienda han experimentado diversas trayectorias de evolución, influidas no solo por las propias transformaciones internas, sino también por el desarrollo y crecimiento global de nuestras ciudades. Esta situación hace necesaria en la actualidad una mirada atenta a 
los problemas y oportunidades que caracterizan de forma específica a cada polígono, más allá de las críticas, a menudo generalistas, sobre los problemas que plantean (Díez Medina y Monclús 2017) y de los manuales de buenas prácticas (García Vázquez et al. 2016).

La dificultad que supone establecer una metodología de diagnóstico que permita entender tanto la evolución del espacio libre como sus oportunidades específicas de regeneración puede ser una de las causas de la carencia de actuaciones de mejora de estos espacios(García-Pérez, Monclús, y Díez Medina 2019a). Por ello, una de las líneas de trabajo del proyecto de investigación UR-Hesp ha sido la elaboración de una metodología híbrida de análisis que permitiera diagnosticar de forma específica la calidad del espacio libre, una aproximación que resulta especialmente útil en los procesos de regeneración urbana integrada durante la toma de decisiones entre los agentes implicados. Además, su aplicación permite, en primer lugar, comparar los diferentes puntos de partida de cada polígono, con el fin de constatar la heterogeneidad en su diseño y el grado de responsabilidad de éste en los resultados; en segundo lugar, analizar las diferentes trayectorias, lo que da lugar a identificar procesos comunes y específicos entre polígonos; finalmente, reconocer aciertos y errores urbanos con la perspectiva que ofrecen cincuenta años de vida, esencial para poder afrontar los retos que plantea su futuro.

Partiendo de trabajos previos que han ayudado a definir esta metodología de análisis y diagnóstico de la calidad del espacio (GarcíaPérez et al. 2019), esta comunicación presenta algunos resultados obtenidos tanto de forma individualizada como comparada, gracias a la aproximación empírica de la investigación. El objetivo es demostrar el potencial que ofrece para ser extrapolada a otros casos de estudio.

\section{Metodología}

El siguiente apartado expone de forma sintética las principales características de la metodología. En primer lugar, UR-Hesp es una metodología híbrida que trabaja con el menor número de variables físicas que ayudan a describir el espacio abierto. Las nueve variables de estudio, relacionadas con los problemas del espacio libre que la literatura previamente ha detectado de forma generalista , están organizadas en base a los tres elementos morfológicos básicos (Oliveira 2016): sistema viario (integración, permeabilidad y elegibilidad), unidades parcelarias (diversidad funcional, espacio intermedio y estructura parcelaria) y edificios (densidad, diversidad edificatoria y diseño a la 'altura de los ojos').

UR-Hesp es una metodología que permite abordar diversas escalas, desde la relación del polígono con la ciudad hasta el fragmento urbano en detalle que considera la relación entre el espacio libre y la edificación. Para cada variable de análisis la metodología supone una particular aproximación a la calidad urbana que combina análisis cuantitativos y cualitativos que permiten evaluarla en términos de 'buena', 'estándar' y 'pobre', con la ayuda de mapas $\mathrm{y}$ diagramas que permiten entender no solo 'cuánta', sino también 'cómo' y 'dónde' se produce dicha calidad (Dovey y Pafka 2017). La metodología tiene en cuenta los procesos urbanos, al considerar tanto su diseño inicial como la situación actual (García-Pérez et al. 2019).

La metodología UR-Hesp se ha ensayado sobre una muestra de 28 polígonos de tres ciudades españolas, representativa de una forma de construir ciudad durante el periodo de posguerra. Su aplicación empírica permite: 1) realizar diagnósticos específicos para cada caso de estudio sobre la calidad del espacio libre que puedan ayudar a la toma de decisiones a los agentes implicados durante procesos de regeneración urbana; 2) reconocer procesos físicos comunes experimentados durante el periodo estudiado y 3) obtener algunas pistas sobre aciertos y errores que ayuden, en clave de futuro, a establecer políticas y estrategias de regeneración urbana de conjuntos. A continuación, se discute el potencial de su aplicación.

\section{Organización de los diagnósticos específicos: de la ficha sistemática a la interpretación}

En primer lugar, este apartado incluye la aplicación metodológica individualizada a 


\section{Barrio de San Antonio de la Florida}
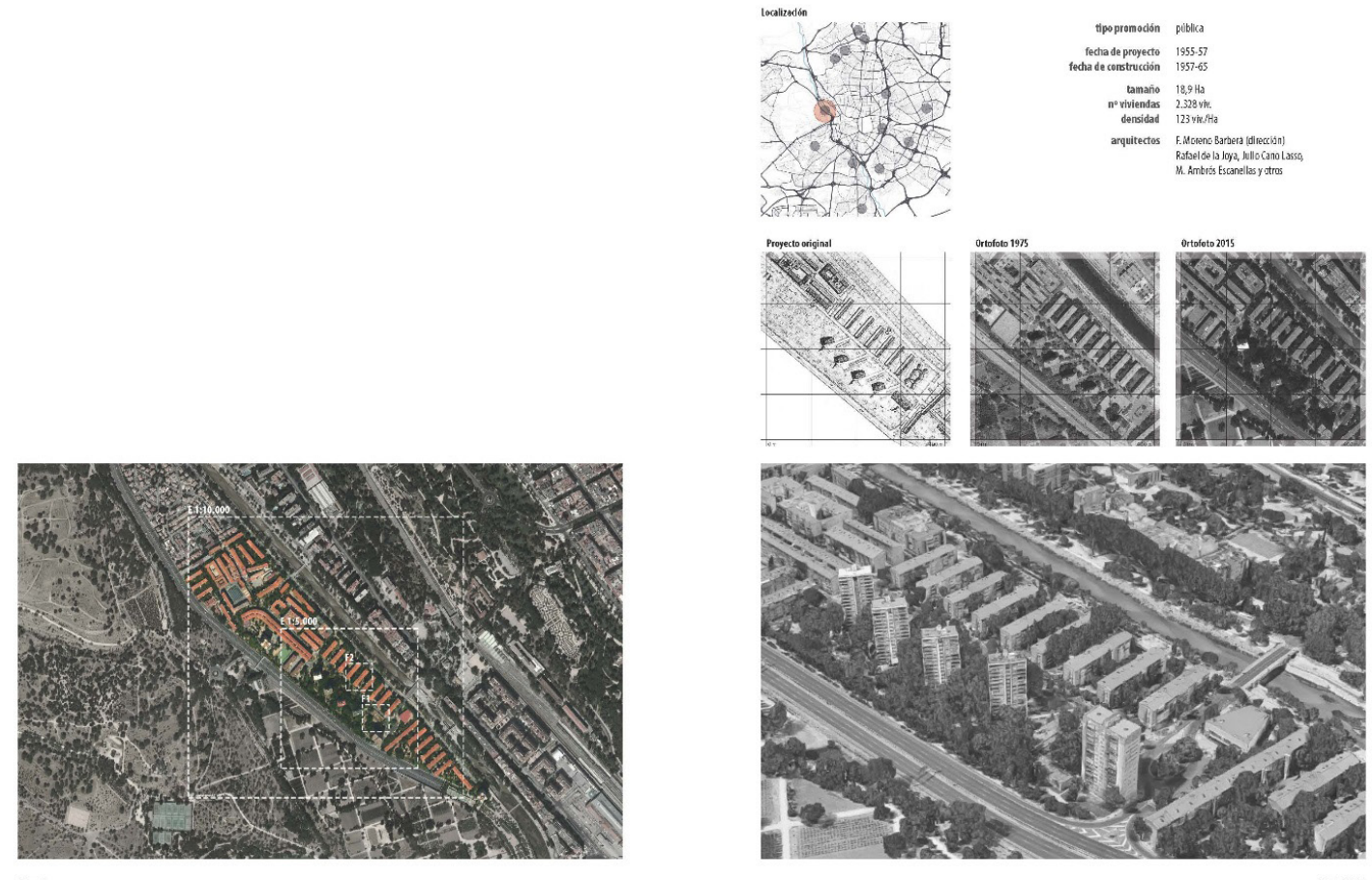

Fig. 1. Ficha de análisis específico. Lámina de introducción

cada caso de estudio. La sistematización de la información obtenida en cada polígono se organiza a través de fichas que ayudan a evaluar la calidad específica de cada uno de los polígonos. Por un lado, se sintetiza la información gráficamente, mediante análisis planimétricos, diagramáticos y fotográficos. Por otro, se procesa esta información en un texto final en el que se interpretan los resultados y se extraen conclusiones. La información que incluye cada ficha se ordena atendiendo a una estructura multiescalar, tal y como se indica a continuación:

- A modo de introducción, en la primera doble página se reúne la información relativa a la localización del polígono y datos generales básicos que ayudan a caracterizarlo (periodo de construcción, tipo de promoción, tamaño $\mathrm{y}$ arquitectos). Esta información se completa con una planta del proyecto original, fotografía aérea histórica y actual y otra oblicua reciente, lo que permite comparar el estado original con la evolución del conjunto (figura 1).

- A continuación, se dedica una página al análisis de los conjuntos a escala de la ciudad y entorno. Aquí se aborda el análisis delas dos primeras variables de calidad en la metodología propuesta: integración con la ciudad y permeabilidad con el entorno. La primera se visualiza con los planos de la evolución de la ciudad entre 1975 y 2015 a escala 1:200.000. Los diagramas de evaluación acompañan al mapa y ofrecen la información sobre la calidad de la variable de estudio. En cuanto a la permeabilidad, los mapas de inserción urbana del polígono a escala 1:20.000 facilitan la comprensión y visualización de la evolución específica experimentada por cada uno de los conjuntos en el periodo estudiado (figura 2).

- La siguiente página presenta en primer lugar un análisis de las variables de probabilidad de elección de la red viaria $\mathrm{y}$, a continuación, un estudio sobre la diversidad tipológica. En primer lugar, la ficha sintetiza la información relevante a la estructura viaria a través del mapa de 'centro de líneas' analizado en Space Syntax, con grosores variables del mayor a menor capacidad de elección en recorridos 

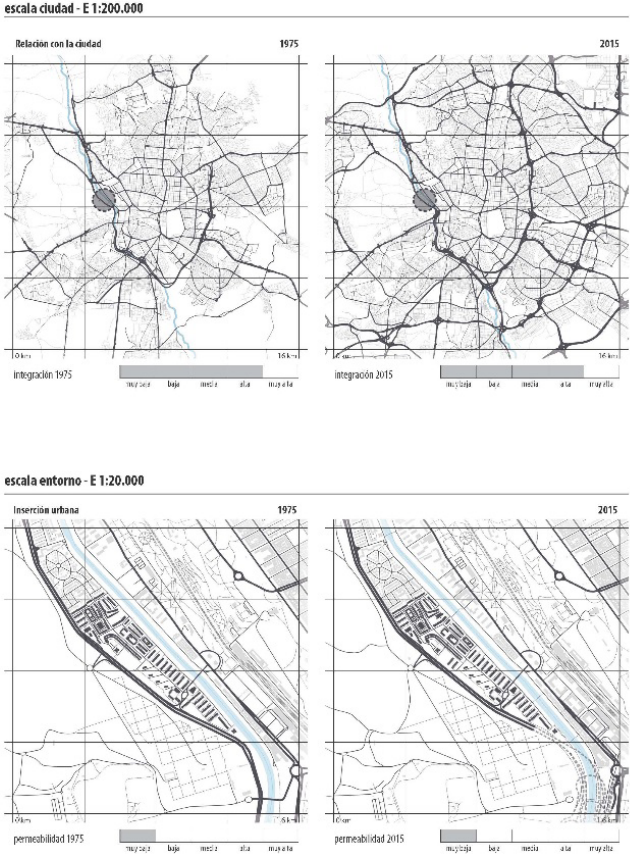

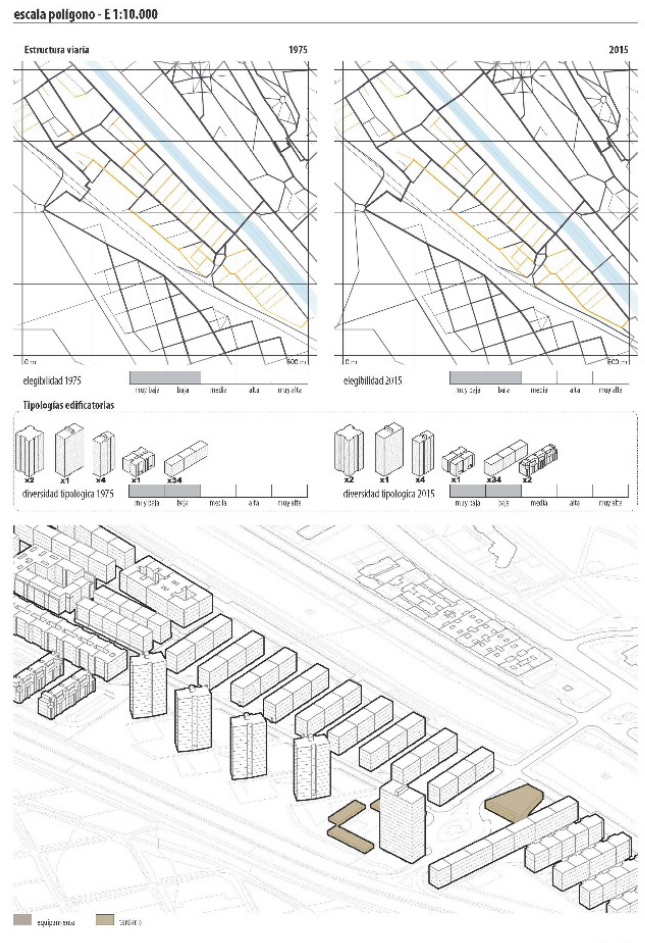

Fig. 2. Ficha de análisis específico. Lámina de escala ciudad, entorno y polígono (I)

locales. El uso del color distingue las vías peatonales de aquellas de uso compartido con vehículos. Los diagramas de evaluación acompañan al mapa y ofrecen información específica sobre la calidad en cada una de las situaciones analizadas. A continuación, la ficha recoge las tipologías edificatorias en un doble gráfico: el primero resume el número y definición de las tipologías edificatorias presentes en el conjunto, así como un diagrama con su valoración; el segundo consiste en una representación tridimensional del conjunto a escala 1:5.000 que facilita la interpretación de los valores de diversidad (figura 2).

- La siguiente doble página aborda el análisis tanto a escala de polígono como de fragmento urbano. La reflexión sobre la escala de polígono se organiza en torno a tres cuestiones: indicadores de densidad, estructura parcelaria y ordenación interna y transformación. Los indicadores de densidad se expresan en el gráfico denominado 'Spacemate' (Berghauser Pont y Haupt 2010), capaz de representar en una sola figura los valores de edificabilidad, ocupación, altura media y adecuación entre el espacio libre y construido. Acompaña a este gráfico una tabla con los valores pormenorizados, incluyendo el indicador de adecuación corregida que relaciona la cantidad de espacio libre de uso público con la superficie construida. El segundo fragmento analiza la estructura parcelaria a través de un mapa del polígono que superpone los límites parcelarios al dibujo del espacio libre a escala 1:5.000. El diagrama clasifica el tipo de estructura parcelaria y añade información relevante sobre la titularidad del espacio libre. El apartado de ordenación interna y transformación mapifica cada polígono a escala 1:5.000. El uso del color sobre la edificación y el suelo permite no solo conocer la cuantía y distribución de cada uno de los usos y clasificaciones establecidas en la metodología, sino también cuantificar su presencia a través de análisis brutos -diagramas edificabilidad y ocupación- y netos - diagrama de evaluación de la diversidad de usos-. Para cada polígono se incluye una valoración sobre la posición y 

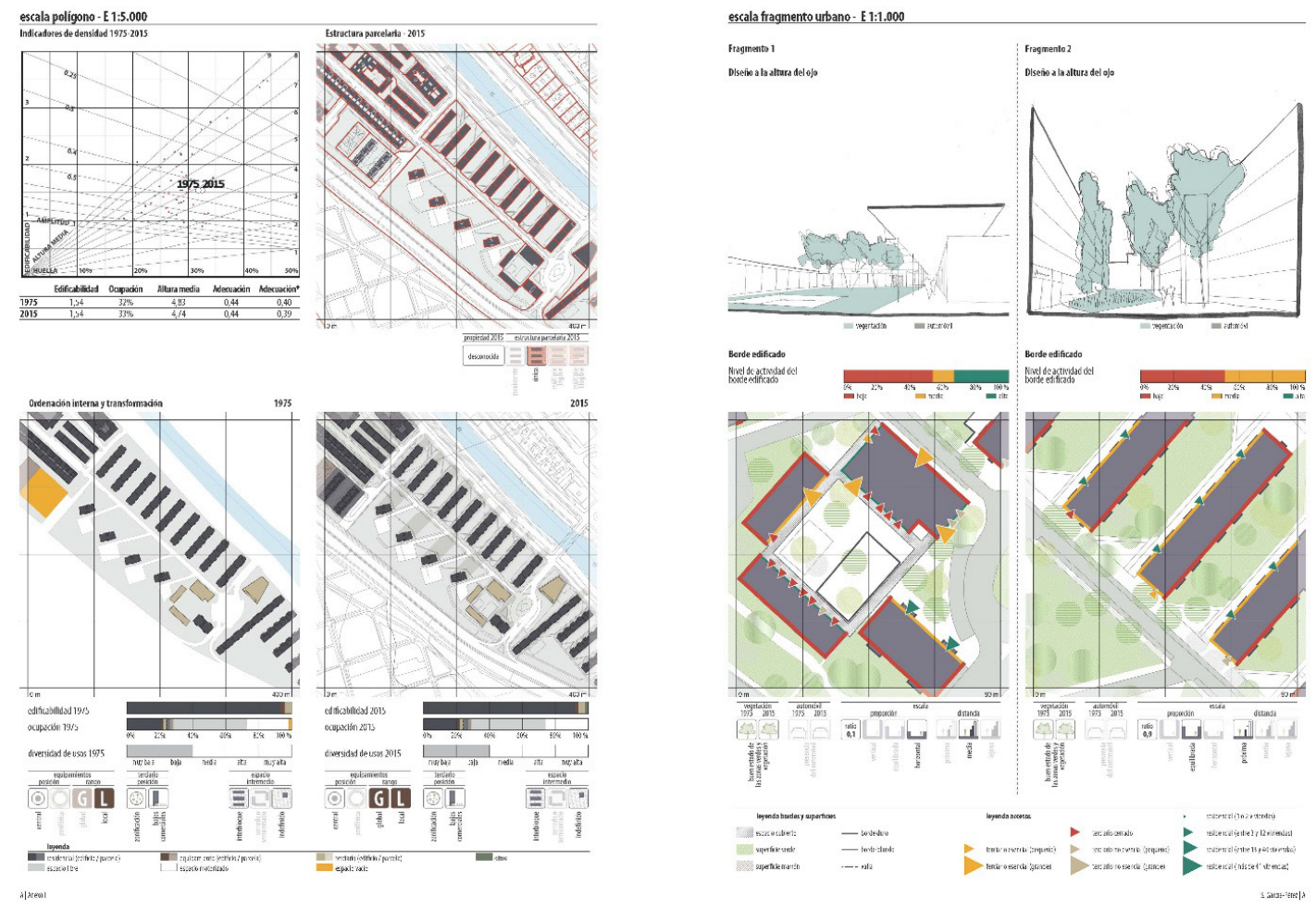

Fig. 3. Ficha de análisis específico. Lámina de escala polígono y fragmentos urbanos

rango de los equipamientos y usos terciarios, así como de los tipos de espacio intermedio. La comparación entre 1975 y 2015 favorece la visualización y cuantificación de procesos de transformación. La siguiente página aborda la escala más pequeña. Para cada uno de estos fragmentos se ha realizado un dibujo en detalle del fragmento objeto de estudio, capaz de capturar la esencia del diseño a la 'altura de los ojos'. Se trata de un dibujo realizado a mano alzada con criterios homogéneos para cada uno de los espacios libres seleccionados. Sobre el dibujo se destaca la situación actual de las zonas verdes y la presencia del automóvil. De forma complementaria, cada uno de estos espacios se mapifican en planta para indicar la actividad del borde edificado -activos, medios e inactivos-, superficies -espacios cubiertos, vegetacióny accesos -según usos y dimensiones-. Por último, para cada fragmento se realiza una valoración final sobre la vegetación, presencia del automóvil y adecuación a la escala humana del espacio (figura 3 ).

- En la última doble página se procesan los análisis anteriores, con el fin de ofrecer una aproximación a la evaluación de la calidad de cada uno de los polígonos. Para ello, se combina un texto crítico con la matriz de evaluación específica. El texto comienza explicando la importancia del polígono analizado y su marco de desarrollo. A continuación, se realiza una breve exposición de los principales problemas socioeconómicos que experimenta, como complemento al análisis físico realizado. Completa este párrafo introductorio un resumen sobre las actuaciones y programas llevados a cabo que ayuden a comprender las transformaciones efectuadas o en curso del polígono. Seguidamente, se justifica la calidad específica en relación con cada una de las variables de estudio. El texto no solo explica los resultados obtenidos, sino que también detecta las causas de las transformaciones analizadas y las oportunidades específicas de cada conjunto. Además, incluye información adicional de interés, que, debido a las propias limitaciones de la metodología, no se explicitan en los gráficos y diagramas. Por último, tras 

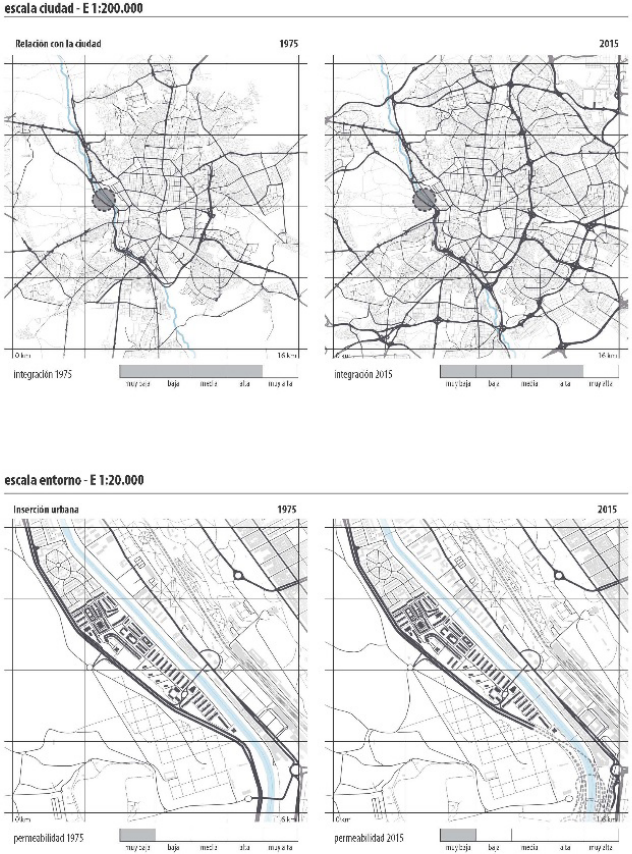

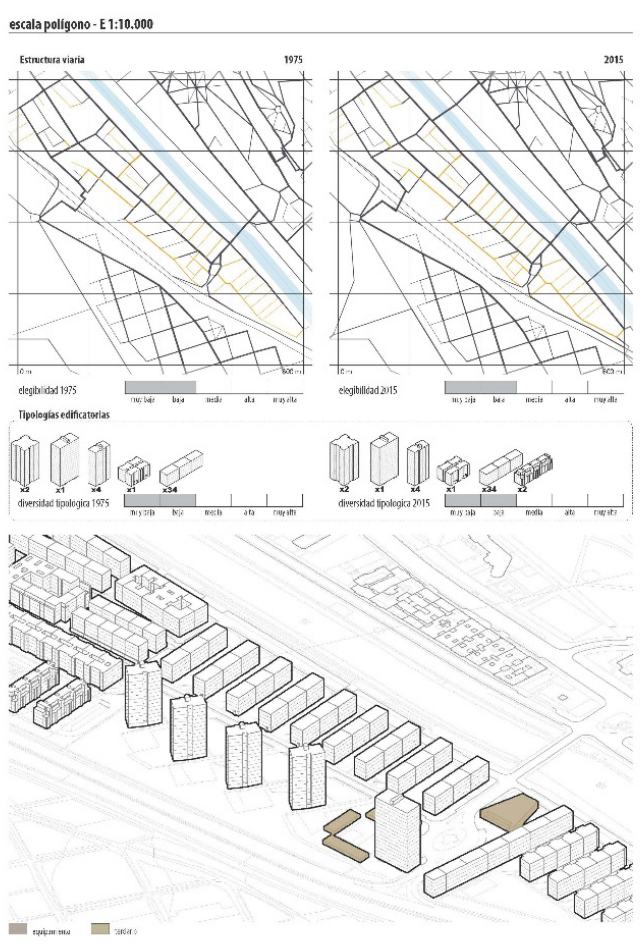

Fig. 4. Ficha de análisis específico. Lámina de texto interpretativo y matriz de valoración de calidad

el diagnóstico efectuado, se sugieren posibles actuaciones de mejora específica que incidan en una mayor calidad del espacio libre (figura 4).

Como se puede observar, la investigación no se ha limitado a la mera recapitulación de información diagramática y gráfica que permitan explicar los procesos físicos experimentados durante los cincuenta años de vida de los polígonos. Más allá de esta visión global, la interpretación de dichos gráficos y diagramas facilita la operatividad de la evaluación y de su representación cartográfica. Así, es posible identificar los problemas y oportunidades que presentan los espacios libres de cada polígono a través de variables que permiten concretar aspectos relacionados con la calidad de los mismos. A través de preguntas tales como 'cuánta', 'cuándo', 'dónde' y 'cómo', en relación con la calidad, se pueden llegar a obtener respuestas bastante acotadas.

\section{Análisis de procesos}

Además del diagnóstico específico de cada uno de los polígonos seleccionados, la sistematización de la información también facilita la interpretación comparada de las variables de estudio. El estudio de los 28 polígonos ha permitido detectar procesos físicos que han favorecido -o no- la mejora de la calidad urbana del espacio público desde una aproximación empírica. A continuación, se exponen de forma sintética los procesos más comunes que el análisis pormenorizado ha permitido detectar:

- Cambios en la ubicación urbana relativa: frente a la situación inicial generalmente periférica de los polígonos, diversos factores -crecimiento de tejidos urbanos, desarrollo de nuevas infraestructuras o mejora de la conectividad de las mismas- han propiciado una 'reubicación' relativa, menos periférica en relación a la estructura urbana.

- Inserción urbana: el aislamiento físico a nivel local ha sido superado gracias a la 
incorporación de nuevas conexiones urbanas a tejidos próximos. Este hecho puede deberse a la transformación interna de los polígonos, a la mejora de sus bordes o a los cambios producidos en los tejidos urbanos próximos. - Diversificación funcional: a menudo se han introducido nuevos usos y servicios en los polígonos, tales como equipamientos (dotación) o actividades comerciales y de ocio (tercerización). Esta diversificación puede producirse o bien por la existencia de reservas de suelo al interior o exterior del polígono, o bien por la transformación de edificaciones ya existentes.

- Aumento de la adecuación corregida: la relación entre la cantidad de espacio libre de uso público y construido ha podido experimentar modificaciones derivadas de transformaciones sobre el espacio abierto (promoción de suelos vacantes, sin uso o aumento de la superficie de estacionamiento), o sobre la edificación (incorporación de nuevas tipologías con o sin actuaciones de renovación urbana).

- Reconfiguración del espacio libre: la incorporación de nuevas tipologías, o bien la renovación y/o transformación de las existentes, ha permitido introducir nuevos arquetipos de espacio intermedio de características diferentes a las anteriores.

- Aumento de la legibilidad de la estructura parcelaria: frente a la falta de correlación entre la estructura parcelaria física y administrativa y debido al desconocimiento de la propiedad del espacio libre en muchos casos, las transformaciones e intervenciones sobre éste han favorecido la introducción de estructuras administrativas más claras.

- Compactación: se ha producido un aumento de la proporción de suelo ocupada por la edificación, que tiene por objeto reajustar la cantidad de espacio libre. Puede responder a modificaciones sobre la edificación (incorporación de nuevos edificios, o aumento de la ocupación de los mismos), o sobre el espacio libre (reducción de la superficie de espacio abierto a favor de otros usos).

- Intensificación: se observa un aumento de la proporción de superficie edificada, que ha tenido por objeto aumentar la co-presencia de agentes y actividades sobre el espacio libre. Al igual que en el proceso anterior, puede deberse a las modificaciones sobre la edificación o el espacio libre.

- Diversificación edificatoria: en algunos casos la finalización de los conjuntos, los procesos de renovación o la incorporación de nuevos edificios residenciales han modificado las condiciones iniciales de riqueza $y$ abundancia de tipologías edificatorias.

Los efectos de estos procesos físicos pueden ser heterogéneos, ya que se comportan de distinta forma en cada uno de los polígonos. A modo de ejemplo, la des-compactación y desintensificación de algunos polígonos puede resultar positiva o negativa, en función de los puntos de partida de cada uno de los polígonos. En el caso de los conjuntos más masivos resulta una estrategia eficaz, mientras que en los más dispersos incrementa los problemas de falta de calidad del espacio libre. Otro ejemplo es la diversificación funcional, cuyo efecto de mejora depende mucho de 'cómo' y 'dónde' se crea la diversidad. Esta reflexión refuerza la necesidad de realizar un diagnóstico de cada polígono desde su especificidad. Aunque los procesos sean comunes, los diferentes puntos de partida y la particular forma de materializar las transformaciones influirán en la trayectoria y calidad actual del espacio libre.

\section{Discusión}

La identificación y estudio de procesos urbanos permite reconocer no solo los errores cometidos durante estos cincuenta años de vida que ahora cumplen muchos de los polígonos, sino también los aciertos que han facilitado la mejora de la calidad urbana en algunos de ellos. En primer lugar, la diversa trayectoria de los polígonos es responsabilidad no solo de los cambios internos que han experimentado, sino también de la propia transformación de la ciudad. Esta reflexión se enmarca en el reconocimiento de la condición orgánica de la ciudad, donde existe una fuerte interrelación entre las 'partes' y el 'todo' (Monclús 2017). La promoción de nuevas áreas de centralidad, los nuevos crecimientos urbanos o el desarrollo de nuevas infraestructuras ejemplifican procesos externos a los polígonos con una incidencia muy alta sobre los mismos, sobre todo en cuestiones como la integración, la inserción 
urbana o la elegibilidad de su estructura viaria.

En segundo lugar, el análisis empírico ha permitido reconocer que la eventual existencia de suelo vacante tanto en el interior como en el exterior de los polígonos ha facilitado en muchos casos la implementación de procesos de transformación. La planificación de reservas de suelo o la disponibilidad de suelo sobre los bordes urbanos ha posibilitado procesos de dotación, generación de espacio libre, intensificación del suelo o una mejor inserción urbana. La no existencia de suelo libre no ha impedido que estas mejoras se produzcan, pero sí ha limitado su alcance.

En tercer lugar, las estrategias de renovación, regeneración o rehabilitación efectuadas en España hasta el momento tienden a no cuestionar la forma urbana de los polígonos de vivienda. En muchos casos, la falta de diagnósticos enfocados en el espacio libre ha dificultado el reconocimiento de las oportunidades y problemas específicos de cada uno de los polígonos. El carácter planificado de los polígonos y la mayor cantidad de espacio libre suponen una gran oportunidad para la promoción de una mayor calidad urbana, el carácter sectorial de las operaciones realizadas, al obviar esta dimensión, no ha optimizado el potencial morfológico de los polígonos de vivienda. Pero, más allá de contemplar esta situación como una pérdida de oportunidad en las intervenciones ya efectuadas, esta reflexión debe entenderse en el marco de un proceso de aprendizaje continuo de las actuaciones llevadas a cabo hasta la fecha. Solo desde la evaluación crítica y continuada de las mismas será posible promocionar aproximaciones eficaces, revisables y adaptables.

Por último, los espacios libres de mayor calidad muestran un adecuado equilibrio entre las variables de estudio seleccionadas. Una correcta interpretación de la calidad exige superar la visión sectorial a favor de otra más integrada. En términos físicos, el éxito del espacio libre de los polígonos de vivienda reside en el equilibrio entre espacios accesibles, bien definidos y conectados, con cierta masa crítica que favorezca la presencia de agentes y actividades sobre el mismo y cuyo diseño sea adecuado a la fisiología del potencial usuario. Es por tanto la suma de variables lo que verdaderamente otorga la calidad a un espacio libre, más allá de aquellos casos que destacan por alguna variable en concreto. Precisamente esta necesidad de calidad equilibrada es lo que dificulta obtener un modelo que reúna todas o casi todas las características en conjunto.

\section{En clave de futuro}

El estudio tanto individualizado como comparado de los 28 casos de estudio a través de la metodología UR-Hesp, así como la identificación de aciertos y errores efectuados en los cincuenta años de vida de los polígonos de vivienda permite apuntar algunas ideas en clave de futuro, anunciadas a continuación:

En primer lugar, es necesario considerar a los polígonos como fragmentos de un tejido urbano más amplio, cuyas oportunidades de mejora deben promoverse a una doble escala, global y local. Las actuaciones en la ciudad pueden tener incidencia sobre los polígonos $\mathrm{y}$ viceversa. Esta perspectiva se alinea con la base de la regeneración urbana integrada que promueve la Declaración de Toledo (EU Ministers for Urban Development 2010).

La contemporaneidad exige asumir que el urbanismo funcionalista es un legado inacabado. Los polígonos de vivienda son, repitiendo las palabras de Druot, Lacaton y Vassal (2007), 'territorios de excepción'. Más allá de los riesgos de una conservación inmovilista o de una excesiva crítica, los polígonos constituyen una gran oportunidad de mejora. Por ello, reconocer y diagnosticar las oportunidades específicas es una tarea primordial, capaz de acercarnos a soluciones de intervención más avanzadas.

Las intervenciones constructivas, sobre todo aquellas enfocadas a la rehabilitación energética y la mejora de la accesibilidad, no deben eclipsar la política de regeneración de los polígonos en el caso español. Una visión integrada valora en el espacio libre una gran oportunidad de reordenación, con amplias posibilidades de reconfiguración, intensificación, revitalización, reintegración y renaturalización. Dada la complejidad de los procesos de declive, de origen sistémico, esta aproximación física desde el espacio público debe considerarse en conjunto a la realidad 
socioeconómica específica del binomio ciudadpolígono.

Las transformaciones internas que generalmente se han llevado a cabo en los polígonos no han sido capaces de favorecer la evolución de los conjuntos. Sin embargo, no hay que olvidar que el diseño urbano tiene la capacidad de promover un nuevo soporte físico en el que dicha evolución sea posible. Se trata, en definitiva, de dotar de herramientas a la forma urbana que permita asumir la incertidumbre y superar la rigidez del espacio libre funcionalista a favor de espacios libres más acordes con la concepción de la 'ciudad abierta' sugerido por Sennett y con las visiones actuales (Sennett 2019).

Tras todo lo expuesto, hay que añadir que mirar retrospectivamente a los contextos en los que surgieron estos los polígonos de vivienda, estudiar cómo fueron diseñados y cómo han evolucionado, resulta de máximo interés. La metodología UR-Hesp es una herramienta de diagnóstico cuya particularidad consiste en aportar datos sobre los procesos pasados y sobre la situación actual específica de los polígonos de vivienda, con una visión comparada y una especificidad que resulta útil para afrontar los retos futuros a los que este importante legado de la modernidad debe hacer frente.

\section{Agradecimientos:}

Este trabajo está financiado por el Ministerio de Economía y Competitividad (BIA201460059-R/ BES 2015/072536), por el grupo de investigación PUPC (Paisajes Urbanos y Proyecto Contemporáneo, PUPC http:// pupc.unizar.es), financiado por el Gobierno de Aragón (T44_17 R) y por el proyecto de investigación MAPZAR (Universidad de Zaragoza, UZ2018-TEC-08).

\section{Notas:}

1 Tejidos urbanos caracterizados por los principios del urbanismo funcionalista, que constituyen gran parte del paisaje residencial de nuestras ciudades durante el crecimiento de posguerra (Wassenberg 2012; Monclús y Díez Medina 2016b).

2 A pesar de la aparente homogeneidad, algunos estudios reconocen el papel clave que juega el diseño urbano inicial en los procesos de obsolescencia y resiliencia de los polígonos (García-Pérez 2018; Wassenberg 2013, 134; Monclús y Díez Medina 2016a)

3 Problemas detectados: aislamiento físico, uso monofuncional, cierta ambigüedad en su densidad/intensidad urbana, espacios semipúblicos y colectivos incontrolables, carácter alienante del entorno físico, ausencia de relación entre los edificios y la calle y falta de urbanización y/o mantenimiento del espacio libre (Moya González 1983; Hall 1997; Turkington, van Kempen, y Wassenberg 2004; García Vázquez et al. 2016).

4 A pesar de la dificultad de definir el término 'calidad', en UR-Hesp se manifiesta una relación indirecta entre los problemas urbanos detectados de forma generalista por la literatura y el diseño urbano específico de cada polígono. Un mejor diseño puede favorecer una mayor inclusividad, vitalidad y seguridad sobre el espacio libre, aunque por sí solo no constituyen una garantía de éxito.

5 Un exceso de patrimonialización de los polígonos puede estancar o empeorar la calidad de los polígonos, al no permitir la evolución y adaptación de las cuestiones más críticas del proyecto de vida moderno. Al contrario, la asociación directa de problemas físicos a sociales puede causar la demolición acrítica de los conjuntos de vivienda, con daños patrimoniales y sociales irreversibles (GarcíaPérez, Monclús, y Díez Medina 2019b).

\section{Referencias}

Berghauser Pont, Meta, y Per Haupt. 2010. Spacematrix: Space, Density and Urban Form. Rotterdam: nai010 publishers.

Díez Medina, Carmen, y Javier Monclús. 2017. "Dealing with mass housing estates legacy: The need of specific diagnoses from an urban design perspective". En Proceedings 24th ISUF 2017 - City and Territory in the Globalization Age, 309-22. Valencia: Universitat Politècnica València. https://doi. org/10.4995/ISUF2017.2017.5887.

Dovey, Kim, y Elek Pafka. 2017. "What is functional mix? An assemblage approach". Planning Theory \& Practice 18 (2): 249-67. 
https://doi.org/10.1080/14649357.2017.128 1996.

Druot, Frédéric, Anne Lacaton, y Jean-Philippe Vassal. 2007. Plus. La vivienda colectiva. Territorio de excepción. Barcelona: Gustavo Gili.

EU Ministers for Urban Development. 2010. "Toledo informal ministerial meeting on urban development declaration Toledo". Toledo.

García-Pérez, Sergio. 2017. "Diseño urbano y espacio público en contextos de regeneración urbana integrada: conceptos, marco institucional y experiencias recientes". ZARCH: Journal of interdisciplinary studies in Architecture and Urbanism 8 (octubre): 214-27. https://doi.org/10.26754/ojs_zarch/ zarch.201782157.

—. 2018. "El proyecto del espacio intermedio de polígonos de vivienda masiva: responsabilidad del diseño en procesos de obsolescencia urbana". En Ciudad y formas urbanas. Perspectivas transversales Volumen 8. Formas urbanas y mapping, editado por Javier Monclús y Carmen Díez Medina, 67-78. Zaragoza: Prensas de la Universidad de Zaragoza e Institución Fernando el Católico. https://doi. org/10.26754/uz.9788417358877.

García-Pérez, Sergio, Javier Monclús, y Carmen Díez Medina. 2019a. "Polígonos de vivienda. Relevancia del diagnóstico en la regeneración urbana de espacios libres". En La Casa. Espacios domésticos, modos de habitar, editado por Juan Calatrava, 681-91. Madrid: Abada.

—. 2019b. "Polígonos de vivienda. Relevancia del diagnóstico en la regeneración urbana de espacios libres". En II Congreso Internacional CULTURA y CIUDAD. La casa: espacios domésticos, modos de habitar, 681-91. Granada: Abada.

García-Pérez, Sergio, Vítor Oliveira, Javier Monclús, y Carmen Díez Medina. 2019. "UR-Hesp: A Methodological Approach for a Diagnosis on the Quality of Open Spaces in Mass Housing Estates". Cities.

García Vázquez, Carlos, Ramón Pico Valimaña, Juan José Sendra Salas, y Elisa Valero Ramos. 2016. Intervención en barriadas residenciales obsoletas. Manual de buenas prácticas. Madrid: Abada.

Hall, Peter. 1997. "Regeneration Policies for Peripheral Housing Estates: Inward- and Outward-looking Approaches". Urban Studies 34 (5-6): 873-90. https://doi. org/10.1080/0042098975862.

Ministerio de Fomento. 2019. "Agenda urbana española 2030". Madrid: Ministerio de Fomento.

Monclús, Javier. 2017. "Entre los CIAM y el urbanismo organicista: tres planes urbanísticos de posguerra (Madrid 1946, Barcelona 1953, Zaragoza 1957)". En actas del Congreso Los años CIAM en España. La otra modernidad, editado por Ricardo Sánchez Lampreave. Madrid: Lampreave.

Monclús, Javier, y Carmen Díez Medina. 2016a. "CIAM Urbanism revisited. Modernist Mass Housing Estates in Spain: Best, Good, Standard, Poor (BGSP)". En Adaptive reuse. The Modern Movement towards the future, 14th International conference proceedings, editado por Ana Tostoes y Zara Ferreira, 779-89. Lisboa: Docomomo internacional Casa da Arquitectura.

. 2016b. "Modernist housing estates in European cities of the Western and Eastern Blocs". Planning Perspectives 31 (4): 533 62. https://doi.org/10.1080/02665433.2015. 1102642.

Monclús, Javier, Carmen Díez Medina, y Sergio García-Pérez. 2017. "Housing estates as urban legacy: urban forms and open spaces / Los polígonos de vivienda como legado urbanístico: formas urbanas y espacios libres". En I Jornadas Periferias Urbanas. La regeneración integral de barriadas residenciales obsoletas, Escuela Técnica Superior de Arquitectura de Sevilla, 22 de febrero de 2017, editado por María Teresa Pérez Cano y Daniel Navas Carrillo, 14-31. Sevilla: Escuela Técnica Superior de Arquitectura Universidad de Sevilla.

Moya González, Luis. 1983. Barrios de promoción oficial. Madrid, 1939-1976. Madrid: Colegio Oficial de Arquitectos de Madrid.

Oliveira, Vítor. 2016. Urban Morphology. The Urban Book Series. Cham: Springer International Publishing. https://doi. org/10.1007/978-3-319-32083-0. 
Sennett, Richard. 2019. Construir y habitar. Ética para la ciudad [Building and dwelling, 2018]. Madrid: Anagrama.

Sotoca, Adolf. 2012. After the Project. Updating Mass Housing Estates. Barcelona: Ediciones UPC.

Turkington, Richard, Ronald van Kempen, y Frank Wassenberg, eds. 2004. High-rise housing in Europe: Current trends and future prospects. Delft: Delft University Press.

United Nations. 2015. "Transforming our world: The 2030 agenda for sustainable development". Resolution adopted by the General Assembly. New York: United Nations General Assembly.

Wassenberg, Frank. 2012. "Housing Estates". En International Encyclopedia of Housing and Home, editado por Susan J. Smith, Marja Elsinga, Lorna Fox O'Mahony, Ong Seow Eng, Susan Wachter, y Pareja Montserrat Eastaway, 3:444-49. Elsevier. https://doi. org/10.1016/B978-0-08-047163-1.00530-0.

. 2013. Large housing estates: Ideas, rise, fall and recovery. The Bijlmermeer and beyond. Amsterdam: IOS Press - Delft University Press. 